\title{
Comparing a Computer-based Childhood Vaccination Registry with Parental Vaccination Cards: A Population-based Study of Delaware Children
}

\author{
Alexander N. Ortega, MPH1,2 \\ Stephen F. Andrews, MS2 \\ Solomon H. Katz, PhD2,3 \\ Steven A. Dowshen, MD2,3 \\ W. Scott Curtice, BA2,3 \\ Mary E. Cannon, PhD2,4 \\ Denice C. L. Stewart, DDS, MHSA2,5 \\ Kyra Kaiser, PhD2
}

Summary: We conducted a population-based study in Delaware to examine the reliability of childhood vaccination data in a comprehensive computer-based record system versus parental vaccination cards. We sampled 1,005 children born between January, 1991, and September, 1993. We oversampled for children whose mothers received Medicaid or were uninsured at the time of delivery. Of the survey responders, $276(56 \%)$ had access to written records, and 409 (83\%) records were located in the Delaware immunization computer database. The kappa coefficient was 0.18. The observed agreement was $59.8 \%$. When the two databases were combined, the up-to-date rate for 2-year-olds was $58.4 \%$, an increase of $12.7 \%$ and $24.2 \%$ from the computer database and the parental records, respectively. The computer database was $78.1 \%$ sensitive and the parental records were $54.9 \%$ sensitive. These results indicate that a comprehensive computer-based record system, with adequate provider participation and proper data management, can be more reliable than parental vaccination cards.

From the 1 University of Michigan School of Public Health, Department of Epidemiology, Ann Arbor; ${ }^{2}$ W. M. Krogman Center for Research in Child Growth and Development, The University of Pennsylvania, Philadelphia; ${ }^{3}$ Alfred I. duPont Institute, Children's Hospital, Wilmington, DE; ${ }^{4}$ Delaware Department of Health and Social Services, Dover, DE; ${ }^{5}$ Dental Care Systems, School of Dental Medicine, The University of Pennsylvania, Philadelphia.

Presented in part at the 30th National Immunization Conference, Washington, D.C., April 11, 1996.

Reprint requests and correspondence to: Alexander N. Ortega, MPH, The W. M. Krogman Center for Research in Child Growth and Development, The University of Pennsylvania, 4019 Irving Street, Philadelphia, PA 19104-6003.

(c) 1997 Westminster Publications, Inc., 708 Glen Cove Avenue, Glen Head, NY 11545, U.S.A.

\section{Introduction}

ollection of immunization
data is important in order
to evaluate compliance, coverage, and practice in various settings. ${ }^{1-9}$ With the initiation of government-led initiatives to increase vaccination completion rates of infants and preschoolaged children, attention needs to be given to the evaluation of the reliability of vaccination data 
sources. The Healthy People 2000 report has pushed for $90 \%$ coverage of four doses of the diphtheria, tetanus, pertussis (DTP) combination vaccine, three doses of the oral polio virus (OPV) vaccine or the inactivated polio virus (IPV) vaccine, and one dose of the measles, mumps, and rubella (MMR) combination vaccine for all 2-year-old children in the United States. ${ }^{10}$ The parental vaccination card is a common source of data used by clinicians, school administrators, and investigators to assess immunization coverage. However, many state health agencies and other health institutions are now considering the advantages of the computerized immunization registry. 11,12 Currently, 22 states and the District of Columbia are using or are in the process of initiating computer-based immunization registries. ${ }^{12}$ Further, since public health agencies use information from immunization coverage assessments to make policy and program-funding decisions, continuous assessment of these tools is necessary.

VacAttack, Delaware's vaccination registry, was established in 1974, which makes it the oldest statewide vaccination registry in the United States. According to the Delaware Division of Public Health's Immunization Program, approximately $74 \%$ of all pediatric and family practice providers in Delaware report data to the registry. In part, this is due to a State policy that requires providers who receive vaccines through the federal Vaccines for Children Program to report to the registry.Vaccinations administered in emergency room departments are also reported to the registry. More than $98 \%$ of all Delaware children have had at least one vaccine dose reported to the registry. This is supported by a
State policy that requires schools and day care centers to report the vaccination histories of all children to the registry, including those born outside the State who have moved to Delaware.

In December 1992, The A. I. duPont Institute Children's Hospital in Delaware, the University of Pennsylvania's Krogman Growth Center, and the Delaware Department of Health \& Social Services (DHSS) initiated a collaborative effort to perform an independent population-based study of vaccination outcomes of 2-year-olds in Delaware as part of a comprehensive child health care survey. By doing so, we were able to examine the reliability of the data collected from parental vaccination cards by comparing them with data from the computer-based record system.

Wilton and Pennisi ${ }^{9}$ have reported that in the UCLA Children's Health Center's computerbased vaccination registry the inaccuracies due to transcription errors made the registry less reliable than medical records. Also Goldstein et al $^{13}$ found that information from vaccination cards was inadequate to accurately assess underimmunization in an emergency room setting. This study attempts to evaluate differences in information from the parental vaccination card and the computerized database in Delaware.

\section{Methods}

The principal tool used to evaluate parental immunization records was a multidimensional child health survey. The survey targeted a representative population of all Delaware children. One thousand and five children born between January 1991 and Sep- tember 1993 were randomly selected through birth registration. To obtain an adequate sample of children of mothers receiving Medicaid or who were uninsured at the time of delivery, we oversampled this population. The oversample consisted of 365 of the total sample of 1,005 children.

In June 1994, surveys were mailed to the parents of these children asking for their participation in the study. Nominally valued incentives were offered for response to the survey. Confirmation calls were made to determine whether the surveys were received and addresses were correct and to encourage participation in the program. In September, a second mailing was sent to those parents who had not returned or completed the survey. Following the second mailing, additional confirmation calls were accompanied by home visits to all the families receiving a second survey. By April 1995, 495 families had responded to the survey. This provided a $68 \%$ response rate, after the exclusion of the families (276) who were determined to have never received the survey. They were excluded on the basis of home visits to the address on the birth certificate, interviews with residents in the neighborhood, and communications with the United States Post Office.

One section of the survey included a series of questions concerning the immunization status of the children. The section asked whether the parents had written records of their children's immunization histories, and if they did not have written records, what their children's immunization statuses were by memory. In this study, $276(56 \%)$ of the 495 parents who returned the survey had access to written records (not from memory) and transcribed 
the immunization dates onto the survey document as requested.

The computer-based immunization registry was then queried for the 495 children whose parents returned the survey. After several checks for aliases and misspellings, $83 \%$ (409) of the children had at least one immunization recorded in the computerbased immunization registry. Table 1 shows the status of both surveillance systems.

Up-to-date (UTD) by 24 months of age is having received 4 DTP, 3 OPV, and 1 MMR. In comparing the UTD status of the children in the Delaware computer-based registry with the parental records, the date that the survey was completed was used as the point of reference. Only vaccination dates that were present in the registry by the date the parent completed the survey were included in the analyses. We estimated from the dates on which the surveys were signed that 243 of the 495 children were at least 24 months of age when the surveys were completed. If we did not have information from the parental vaccine records or the computer-based registry, it was assumed for our study purposes that no immunizations were administered to that child. Some parents may have had vaccine records but chose not to report the vaccinations on the survey. This is unlikely, however, because of our aggressive follow-up of all subjects.

\section{Data Analysis}

The data were analyzed by the following methods. The kappa statistic was used to measure the interrater reliability between the parental vaccine records and the computerized registry database. A

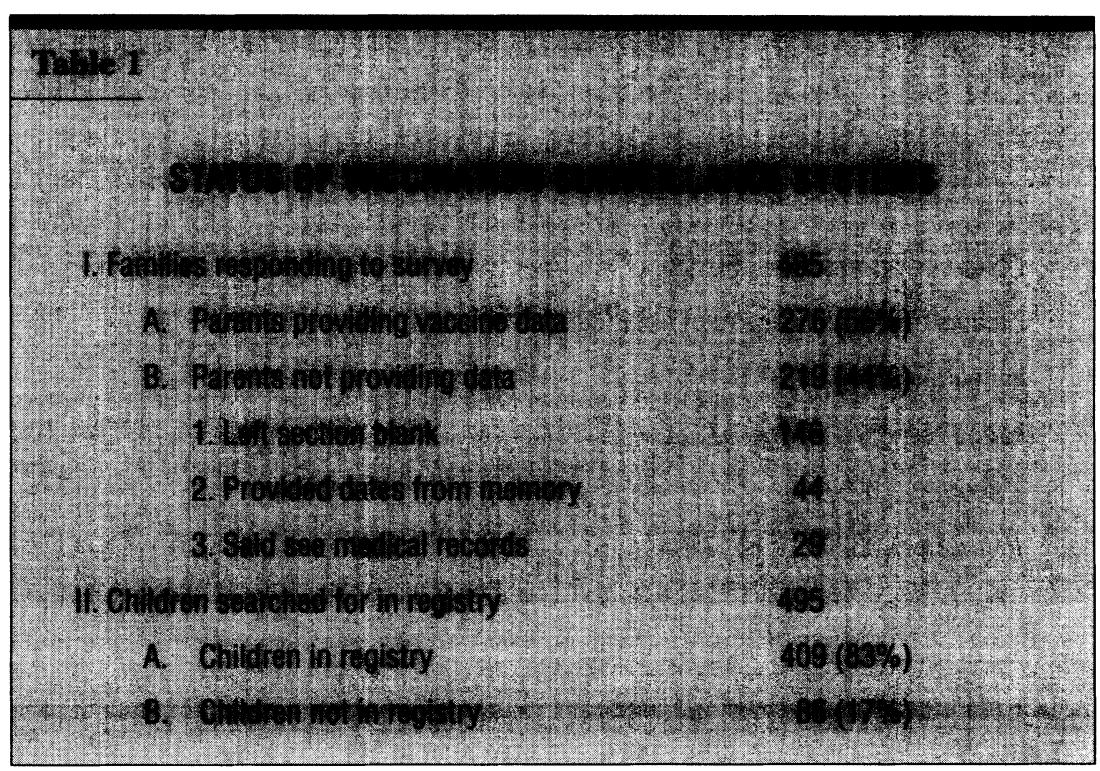

gold standard was created by combining the immunization histories from the computer-based registry with the records provided by the childrens' parents. This composite database was created by reviewing the two databases on a child-by-child basis and combining the two when appropriate. Immunization rates were then calculated for the three surveillance systems: parental records, the Delaware computer-based registry, and the composite immunization database. Finally, we looked at the sensitivity of the parental records and the computer-based registry versus the composite immunization database. This was done in order to compare the performance of the two as screening tools.

\section{Results}

The kappa coefficient was calculated and can be used to assess the agreement of two systems accounting for agreement due to chance. Typically, kappa values greater than 0.75 may be taken to represent excellent agreement beyond chance, values below 0.40 represent poor agreement beyond chance, and values between the two are considered fair to good.14,15 The kappa coefficient was 0.18 for the parental records and the computer-based records which reported immunizations for the same children. The observed agreement between the two systems was $59.8 \%$, and the chance expected agreement was $51.2 \%$ (Table 2). Note that the observed agreement between the two was not highly associated with the children's health care coverage. The observed agreement between the registry and the parental records was $62.5 \%$ and $59.5 \%$ for children whose health care was covered by Medicaid and commercial insurance, respectively. These two types of coverage represent $84 \%$ of the children who were at least 24 months of age when the survey was returned. The uninsured had a lower observed agreement, $50.0 \%$, but these children represent only $12.3 \%$ of the survey population. 

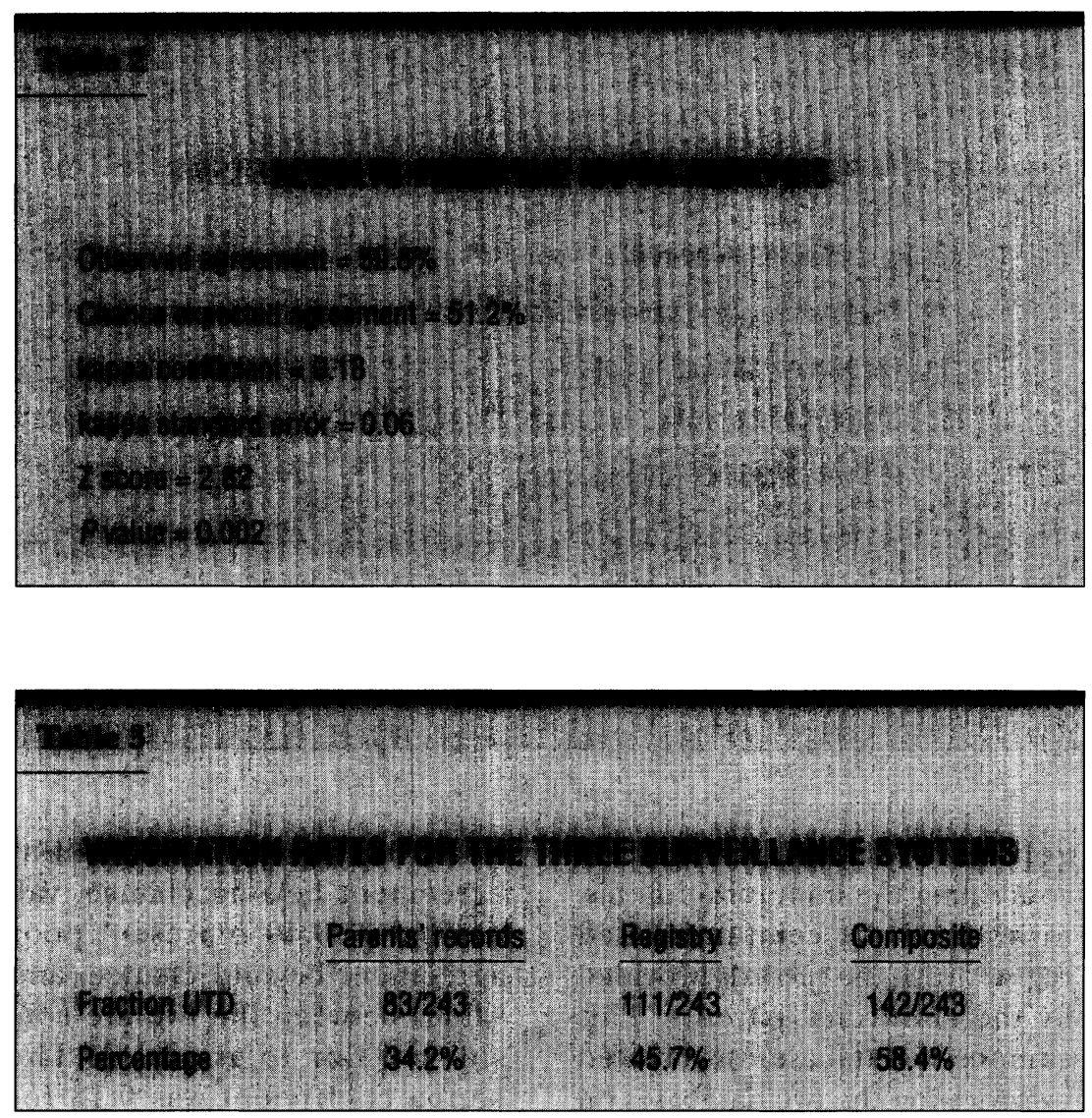

When the immunization histories from the parental records and the computer-based registry were combined, $58.4 \%$ of the children were determined to be UTD by 24 months of age. This shows an increase of $12.7 \%$ from the computer database and an increase of $24.2 \%$ from the parental vaccination cards (Table 3).

When we evaluated the sensitivity of the databases we found that the computer-based record system had a sensitivity of $78.1 \%$ $(111 / 142)$, and the sensitivity of the parental vaccine records was $54.9 \%$ (78/142), when comparing them with the composite. This means that if a child is found to be UTD in the composite, he or she has a $78.1 \%$ probability of being UTD according to the registry or a $54.9 \%$ probability according to the vaccination cards.

\section{Discussion}

This is the first populationbased study that has compared vaccination data in a statewide computerized vaccination registry and parental vaccine cards. We have found that data from a statewide immunization registry are more reliable than parental vaccine cards. The computerbased record system was $23.2 \%$ more sensitive than the parental vaccine records. In addition, only $56 \%$ of the children had at least one immunization reported in the parental vaccination cards, while $83 \%$ had a recorded vaccination in the computer database. Considering a kappa coefficient of 0.18 and a combined UTD rate of $58.4 \%$, it follows that a method that combines both systems to determine immunization rates will increase the reliability of the data. Since this is not practical for many health agencies, the best alternative is to utilize the computer database. Thus, our research supports the establishment of a comprehensive statewide or a nationwide immunization registry.

Data in vaccination surveillance systems are skewed for various reasons. For example, parental vaccination cards are likely to be inaccurate when parents fail to bring their children's records to each office or clinic visit when a vaccine is administered. ${ }^{13}$ The registry is also limited when providers do not report vaccinations or when transcription errors occur. ${ }^{9}$ Our results show that $92.2 \%(212 / 230)$ of the children whose health care was covered by Medicaid or were uninsured when the survey was completed were located in the registry. However, only $73.1 \%$ $(182 / 249)$ of the commercially insured children were in the registry. This clearly demonstrates the effectiveness of state-funded incentives for reporting vaccinations. It should be realized that these percentages are not the actual reporting rates of providers. A child may have some entries in the registry, but all doses of vaccines administered may not have been reported by the provider. In addition, vaccines are reported by schools and day care centers to the registry.

It is apparent that much work needs to be done to improve the information in both systems. It is important to increase the reliability of the information reported to health agencies so that reliable estimates of population coverage can be determined in order to make sound policy decisions and plan intervention strategies. Providers can decrease the number of missed vaccination opportunities by more 
accurately identifying and/or tracking those individual children who are not up-to-date with the vaccination series. This can be facilitated by utilizing a statewide registry, where vaccinations for a child can be assessed by whoever provides care for him or her.

Agencies that are planning and establishing computer-based vaccination registries should take into account problems that have existed in other states. One of the important problems that needs to be addressed is information access and confidentiality. This has been extensively reviewed by Gostin and Lazzarini. ${ }^{12}$ Providers and parents need access to the information in the registry in order to make full use of the system. This could be accomplished by accessing the Wide Area Network or other Internet programs and by assigning passwords to appropriate personnel.

Our research has limitations that need to be addressed. Gathering medical records that may have additional information that is not included in the parental vaccination cards or the registry was not practical at this time owing to cost and time constraints. The other obvious limitation is generalizability. Because Delaware's population size is relatively small, we cannot assert that these results are generalizable to larger states or local regions. In addition, we do not know the status of the immunization histories of the $146(29 \%)$ parents who left the question blank, the $44(9 \%)$ who provided dates from memory, or the $29(6 \%)$ who wrote "see medical records." It is likely that these parents did not have written records since they completed the rest of the survey. Furthermore note that VacAttack is the oldest registry in the United States, and registries take time to mature.
Newer registries may not have had the time to develop the same level of comprehensiveness.

An effectively designed and utilized computer-based record system should also implement computer-generated telephone or postcard reminders, which is an effective intervention strategy. ${ }^{16}$ With adequate provider participation and quality control, the computer database will assist public health officials and providers in targeting populations of underimmunized children more effectively. The results demonstrate the need for agencies and institutions to support the development of computer-based registries in order to obtain more reliable immunization data by distinguishing between those children who are truly underimmunized versus those whose immunizations have been underreported. Such a tracking system would improve the effectiveness of immunization policies and intervention strategies.

\section{REFERENCES}

1. Centers for Disease Control and Prevention. National, state, and urban area vaccination coverage levels among children aged 19-35 monthsUnited States, April 1994-March 1995. MMWR Morb Mortal Wkly Rep. 1996;45:145-150.

2. Centers for Disease Control and Prevention. Use of a data-based approach by a health maintenance organization to identify and address physician barriers to pediatric vaccination-California, 1995. MMWR. Morb Mortal Wkly Rep. 1996;45:188-193.

3. Dietz VJ, Stevenson J, Zell E, et al. Potential impact on vaccination coverage levels by administering vaccine simultaneously and reducing dropout rates. Arch Pediatr Adolesc Med. 1994;148:943-949.

4. Farizo KM, Stehr-Green PA, Markowitz LE, Patriarca PA. Vaccination levels and missed opportunities for measles vaccination: a record audit in a public pediatric clinic. Pediatrics. 1992;89:589-592.
5. Frank RG, Dewa CS, Holt E, et al. The demand for childhood immunizations: results from the Baltimore immunization study. Inquiry. 1995;32: 164-173.

6. Holt E, Guyer B, Hughart N, et al. The contribution of missed opportunities to childhood underimmunization in Baltimore. Pediatrics. 1996;97:474-480.

7. Payne TH. Measuring and improving immunization rates at Group Health Cooperative of Puget Sound. Quality Matters of the National Committee for Quality Assurance. Summer 1995;II:4-6.

8. Szilagyi PG, Rodewald LE, Humiston SG, et al. Missed opportunities for childhood vaccinations in office practices and the effect on vaccination status. Pediatrics. 1993;91:1-7.

9. Wilton R, Pennisi AJ. Evaluating the accuracy of transcribed computerstored immunization data. Pediatrics. 1994;94:902-906.

10. Public Health Service. Healthy People 2000: National Health Promotion and Disease Prevention Objectives. Washington DC: US Department of Health and Human Services, Public Health Service, 1991; DHHS publication no. (PHS) 91-30213.

11. Cutts FT, Zell ER, Mason D, et al. Monitoring progress toward US preschool immunization goals. JAMA. 1992;267: 1952-1955.

12. Gostin LO, Lazzarini Z. Childhood immunization registries. A national review of public health information systems and the protection of privacy. JAMA. 1995;274:1793-1799.

13. Goldstein KP, Kviz FJ, Daum RS. Accuracy of immunization histories provided by adults accompanying preschool children to a pediatric emergency department. JAMA. 1993;270:2190-2194.

14. Fleiss JL. Statistical Methods of Rates and Proportions, 2nd ed. New York: Wiley; 1981.

15. Dawson-Saunders B, Trapp RG. Basic and Clinical Biostatistics. Norwalk, CT: Appleton and Lange; 1990.

16. Stehr-Green PA, Dini EF, Lindegren ML, Patriarca PA. Evaluation of telephoned computer-generated reminders to improve immunization coverage at inner-city clinics. Public Health Reports. 1993;108:426-430. 